\title{
INJECTIVITY OF OPERATOR SPACES
}

\author{
ZHONG-JIN RUAN
}

\begin{abstract}
We study the structure of injective operator spaces and the existence and uniqueness of the injective envelopes of operator spaces. We give an easy example of an injective operator space which is not completely isometric to any $C^{*}$-algebra. This answers a question of Wittstock [23]. Furthermore, we show that an operator space $E$ is injective if and only if there exists an injective $C^{*}$-algebra $A$ and two projections $p$ and $q$ in $A$ such that $E$ is completely isometric to $p A q$.
\end{abstract}

\section{INTRODUCTION}

Let $E$ be a vector space over the complex numbers $C$ and $M_{n}(E)$ the space of $n \times n$ matrices with entries in $E$. We write

$$
x \oplus y=\left[\begin{array}{ll}
x & 0 \\
0 & y
\end{array}\right] \in M_{n+m}(E)
$$

and

$$
\alpha x=\left[\sum_{j=1}^{n} \alpha_{i j} x_{j k}\right] \text { and } x \alpha=\left[\sum_{j=1}^{n} x_{i j} \alpha_{j k}\right] \in M_{n}(E)
$$

for $x=\left[x_{i j}\right] \in M_{n}(E), y=\left[y_{i j}\right] \in M_{m}(E)$ and $\alpha=\left[\alpha_{i j}\right] \in M_{n}(C)$.

An m.n. space (matricially normed space) is a vector space $E$ together with a norm \|\|$_{n}$ on each $M_{n}(E)$, which satisfies:

(i) $\|x \oplus 0\|_{n+m}=\|x\|_{n}$,

(ii) $\|\alpha x\|_{n} \leq\|\alpha\|\|x\|_{n}$ and $\|x \alpha\|_{n} \leq\|x\|_{n}\|\alpha\|$

for all $\alpha \in M_{n}(C), x \in M_{n}(E)$ and the zero element $0 \in M_{m}(E)$. We use the notation $\left(E,\left\{\|\|_{n}\right\}\right)$, or simply $E$. An m.n. space is $L^{\infty}$ if it satisfies

$$
\left(L^{\infty}\right) \quad\|x \oplus y\|_{n+m}=\max \left\{\|x\|_{n},\|y\|_{m}\right\} .
$$

An m.n. space is $L^{p} \quad(1 \leq p<\infty)$ if it satisfies

$$
\left(L^{p}\right) \quad\|x \oplus y\|_{n+m}=\left(\|x\|_{n}^{p}+\|y\|_{m}^{p}\right)^{1 / p} .
$$

Remark. Notice that our definition of m.n. spaces is different from that in Effros [5] and that in Wittstock [23]. According to our notation, the matricially normed

Received by the editors October 1, 1987 and, in revised form, March 2, 1988.

1980 Mathematics Subject Classification (1985 Revision). Primary 46L05.

The author was supported by the Sloan Foundation. 
space defined in [5] is a vector space with a norm \|\|$_{n}$ on each $M_{n}(E)$, and the matricial normed space defined in [23] is an $L^{\infty}$-m.n. space.

Let $E$ and $F$ be m.n. spaces and $\phi: E \rightarrow F$ a linear map. We can define a linear map $\phi_{n}: M_{n}(E) \rightarrow M_{n}(F)$ by letting

$$
\phi_{n}\left(\left[x_{i j}\right]\right)=\left[\phi\left(x_{i j}\right)\right]
$$

for $\left[x_{i j}\right] \in M_{n}(E)$. Write

$$
\|\phi\|_{c b}=\sup \left\{\left\|\phi_{n}\right\| ; n \in N\right\} .
$$

We call $\phi$ a completely bounded map if $\|\phi\|_{c b}<\infty$, a complete contraction if $\|\phi\|_{c b} \leq 1$ and a complete isometry if each $\phi_{n}$ is an isometry.

Let $B(H)$ be the space of all bounded linear operators on a Hilbert space $H$ with the operator norm defined by

$$
\|x\|=\sup \{\|x \xi\| ; \xi \in H,\|\xi\| \leq 1\} .
$$

Identifying $M_{n}(B(H))$ with $B\left(H^{n}\right)$ where $H^{n}=H \oplus \cdots \oplus H$, we obtain a natural operator norm on each $M_{n}(B(H))$. This family of norms $\left\{\|\|_{n}\right\}$ over $B(H)$ is called an operator matricial norm on $B(H)$. A linear subspace of $B(H)$ with the above operator matricial norm is called a concrete operator space. An m.n. space $E$ is called an abstract operator space, or simply an operator space if $E$ is completely isometric to a concrete operator space.

It is obvious that operator spaces are $L^{\infty}$-m.n. spaces. Ruan [18] showed that an m.n. space $E$ is an operator space iff $E$ is an $L^{\infty}-$ m.n. space. This gives a matricial norm characterization of operator spaces. For our convenience, we will regard the words "operator spaces" and " $L^{\infty}-$ m.n. space" as the same notation.

In classical functional analysis, we are interested in studying the properties of normed (Banach) spaces and bounded linear maps between these spaces. It is known that every normed (Banach) space can be canonically embedded into $C(\Omega)$, where $\Omega$ is a compact Hausdorff space and $C(\Omega)$ is the space of all continuous functions on $\Omega$. If $\Omega$ is a stonean space (cf. $[2, \S 7]$ ), then $C(\Omega$ ) is an injective Banach space. Furthermore, Nachbin [14], Goodner [7] and Kelley [12] (Hasumi [10]) have proved that a real (complex) Banach space is injective if and only if it is linearly isometric to $C(\Omega)$ for some stonean space $\Omega$. The existence and uniqueness of the injective envelopes of Banach spaces were studied by Cohen [4].

In quantized functional analysis, we consider operator spaces and completely bounded maps between these spaces. The Arveson-Wittstock Hahn-Banach Theorem has shown that $B(H)$ is an injective object in the category of operator spaces and complete contractions, i.e. $B(H)$ is an injective operator space. Thus every injective $C^{*}$-algebra is an injective operator space. Choi and Effros [3] showed that an operator system is injective in the category of operator systems and completely positive maps if and only if it is completely order isomorphic to an injective $C^{*}$-algebra. In [23], Wittstock asked a question: "Does there 
exist an injective operator space (i.e. an injective $L^{\infty}$-m.n. space), which is not completely isometric to an injective $C^{*}$-algebra?"

In this paper, we study the structure of injective operator spaces and the unique existence of the injective envelopes of operator spaces. We give an example of an injective operator space which is not completely isometric to any $C^{*}$-algebra (Theorem 4.3 ). This gives a negative answer to Wittstock's question. In general, an injective operator space is a triple subsystem of a $C^{*}$ algebra (cf. Youngson [24]). Indeed, we show in Theorem 4.5 that an operator space $E$ is injective if and only if there exists an injective $C^{*}$-algebra $A$ and two projections $p$ and $q$ such that $E$ is completely isometric to $p A q$. Finally we show in $\S 5$ that for every operator space $E$, there exists an essentially unique injective envelope of $E$. Our proof of this result is inspired by Hamana [9].

This paper is a part of my Ph.D dissertation at UCLA. I wish to express my deepest gratitude to my advisor, Professor Edward G. Effros, for his guidance and encouragement throughout this work. I also wish to thank the referee for his many valuable suggestions. Finally, I want to acknowledge that this research was supported by the Alfred P. Sloan Foundation, Grant DD-100.

\section{MATRICIALly SEMINORMED SPACES}

Definition 2.1. Let $E$ be a vector space and $p_{n}$ a seminorm on $M_{n}(E) \quad(n \in$ $N) .\left(E,\left\{p_{n}\right\}\right)$ is called an m.s.n. space (matricially seminormed space) if it satisfies the conditions (i) and (ii) in $\S 1$, i.e.

(i) $p_{n+m}(x \oplus 0)=p_{n}(x)$,

(ii) $p_{n}(\alpha x) \leq\|\alpha\| p_{n}(x)$ and $p_{n}(x \alpha) \leq\|\alpha\| p_{n}(x)$

for all $\alpha \in M_{n}(C), x \in M_{n}(E)$ and 0 the zero element in $M_{m}(E)$. The family of seminorms $\left\{p_{n}\right\}$ is called a matricial seminorm on $E$. An m.s.n. space is called an $L^{p}$-m.s.n. space $(1 \leq p \leq \infty)$ if it satisfies the $L^{p}$-condition.

Proposition 2.2. Let $\left(E,\left\{p_{n}\right\}\right)$ be an m.s.n. space and $K_{n}=\operatorname{ker} p_{n}$, the kernel of $p_{n}$. Then $K_{n}=M_{n}\left(K_{1}\right)$ for each $n \in N$.

Proof. Let $\left[x_{i j}\right] \in K_{n}$. Then

$$
p_{1}\left(x_{i j}\right)=p_{1}\left([0 \cdots 1 \cdots 0]\left[x_{i j}\right]\left[\begin{array}{c}
0 \\
\vdots \\
1 \\
\vdots \\
0
\end{array}\right]\right) \leq p_{n}\left(\left[x_{i j}\right]\right)=0 \text {. }
$$

Hence $p_{1}\left(x_{i j}\right)=0$ and $x_{i j} \in K_{1}$.

Conversely if $x_{i j} \in K_{1}$, we have $p_{n}\left(\left[x_{i j}\right]\right) \leq \sum_{i, j=1}^{n} p_{1}\left(x_{i j}\right)=0$ and thus $\left[x_{i j}\right] \in K_{n}$. Therefore $K_{n}=M_{n}\left(K_{1}\right)$.

Identifying $M_{n}\left(E / K_{1}\right)$ with $M_{n}(E) / K_{n}$, we may define a norm \|\|$_{p_{n}}$ on each $M_{n}\left(E / K_{1}\right)$ by letting

$$
\left\|\left[\bar{x}_{i j}\right]\right\|_{p_{n}}=p_{n}\left(\left[x_{i j}\right]\right)
$$


where $\bar{x}_{i j}$ is the corresponding equivalence class in $E / K_{1}$. It is easy to see that the quotient space $E / K_{1}$ with the matricial norm defined above is an m.n. space. Furthermore if $\left(E,\left\{p_{n}\right\}\right)$ is an $L^{p}$-m.s.n. space, then $\left(E / K_{1},\left\{\|\|_{p_{n}}\right\}\right)$ is an $L^{p}$-m.n. space.

Suppose that $E$ and $F$ are two m.n. spaces and $\phi: E \rightarrow F$ is a complete contraction.

Example 2.3. For each $n \in N$, we define a seminorm $p_{n}^{\phi}$ on $M_{n}(E)$ by

$$
p_{n}^{\phi}(x)=\left\|\phi_{n}(x)\right\|_{n}
$$

for $x \in M_{n}(E)$. Then $\left(E,\left\{p_{n}^{\phi}\right\}\right)$ is an m.s.n. space with $p_{n}^{\phi}(x) \leq\|x\|_{n}$ for all $x \in M_{n}(E)$. If $F$ is an $L^{p}$-m.n. space, then $\left(E,\left\{p_{n}^{\phi}\right\}\right)$ is an $L^{p}$-m.s.n. space.

Proof. It follows easily from the definition.

Example 2.4. Suppose that $F$ is an m.n. subspace of $E$. For each $n \in N$, we define a seminorm $q_{n}^{\phi}$ on $M_{n}(E)$ by

$$
q_{n}^{\phi}(x)=\lim _{k \rightarrow \infty} \sup \left\|\left(\frac{\phi_{n}+\cdots+\phi_{n}^{k}}{k}\right)(x)\right\|_{n}
$$

for $x \in M_{n}(E)$. Then $\left(E,\left\{q_{n}^{\phi}\right\}\right)$ is an m.s.n. space with $q_{n}^{\phi}(x) \leq\|x\|_{n}$ for all $x \in M_{n}(E)$. If $E$ is an $L^{\infty}$-m.n. space, then $\left(E,\left\{q_{n}^{\phi}\right\}\right)$ is an $L^{\infty}$-m.s.n. space. Proof. Given $x \in M_{n}(E)$ and 0 the zero element in $M_{m}(E)$, we have

$$
\begin{aligned}
q_{n+m}^{\phi}(x \oplus 0) & =\lim _{k \rightarrow \infty} \sup \left\|\left(\frac{\phi_{n+m}+\cdots+\phi_{n+m}^{k}}{k}\right)(x \oplus 0)\right\|_{n+m} \\
& =\lim _{k \rightarrow \infty} \sup \left\|\left(\frac{\phi_{n}+\cdots+\phi_{n}^{k}}{k}\right)(x) \oplus\left(\frac{\phi_{m}+\cdots+\phi_{m}^{k}}{k}\right)(0)\right\|_{n+m} \\
& =\lim _{k \rightarrow \infty} \sup \left\|\left(\frac{\phi_{n}+\cdots+\phi_{n}^{k}}{k}\right)(x)\right\|_{n}=q_{n}^{\phi}(x) .
\end{aligned}
$$

For $x \in M_{n}(E)$ and $\alpha \in M_{n}(C)$, we have

$$
\begin{aligned}
q_{n}^{\phi}(\alpha x) & =\lim _{k \rightarrow \infty} \sup \left\|\left(\frac{\phi_{n}+\cdots+\phi_{n}^{k}}{k}\right)(\alpha x)\right\|_{n} \\
& \leq\|\alpha\| \lim _{k \rightarrow \infty} \sup \left\|\left(\frac{\phi_{n}+\cdots+\phi_{n}^{k}}{k}\right)(x)\right\|_{n} \\
& =\|\alpha\| q_{n}^{\phi}(x) .
\end{aligned}
$$

Similarly, we have $q_{n}^{\phi}(x \alpha) \leq\|\alpha\| q_{n}^{\phi}(x)$. Hence $\left(E,\left\{q_{n}^{\phi}\right\}\right)$ is an m.s.n. space. If $E$ is an $L^{\infty}$-m.n. space, then $F$ is also an $L^{\infty}$-m.n. space. For $x \in M_{n}(E)$ 
and $y \in M_{m}(E)$, we have

$$
\begin{aligned}
q_{n+m}^{\phi}(x \oplus y) & =\lim _{k \rightarrow \infty} \sup \left\|\left(\frac{\phi_{n+m}+\cdots+\phi_{n+m}^{k}}{k}\right)(x \oplus y)\right\|_{n+m} \\
& =\lim _{k \rightarrow \infty} \sup \left\{\max \left\{\left\|\left(\frac{\phi_{n}+\cdots+\phi_{n}^{k}}{k}\right)(x)\right\|_{n},\left\|\left(\frac{\phi_{m}+\cdots+\phi_{m}^{k}}{k}\right)(y)\right\|_{m}\right\}\right\} \\
& =\max \left\{\lim _{k \rightarrow \infty} \sup \left\|\left(\frac{\phi_{n}+\cdots+\phi_{n}^{k}}{k}\right)(x)\right\|\left\|_{n}, \lim _{k \rightarrow \infty} \sup \right\|\left(\frac{\phi_{m}+\cdots+\phi_{m}^{k}}{k}\right)(y) \|_{m}\right\} \\
& =\max \left\{q_{n}^{\phi}(x), q_{m}^{\phi}(y)\right\} .
\end{aligned}
$$

Hence $\left(E,\left\{q_{n}^{\phi}\right\}\right)$ is an $L^{\infty}$-m.s.n. space.

Now we consider the Arveson-Wittstock Hahn-Banach Theorem for $L^{\infty}$ m.s.n. spaces.

Theorem 2.5. Let $\left(E,\left\{p_{n}\right\}\right)$ be an $L^{\infty}$-m.s.n. space, $\left(F,\left\{p_{n}\right\}\right)$ an $L^{\infty}$-m.s.n. subspace of $E$ and $\phi: F \rightarrow B(H)$ a linear map such that $\left\|\phi_{n}(x)\right\|_{n} \leq p_{n}(x)$ for all $x \in M_{n}(F)$ and $n \in N$. Then there exists a linear map $\bar{\phi}: E \rightarrow B(H)$ which extends $\phi$ and $\left\|\bar{\phi}_{n}(x)\right\|_{n} \leq p_{n}(x)$ for all $x \in M_{n}(E)$.

Proof. Let $K_{1}=\operatorname{ker} p_{1}$. Then $\left(E / K_{1},\left\{\|\|_{p_{n}}\right\}\right)$ is an $L^{\infty}$-m.n. space. By linear algebra, we may identify $F /\left(F \cap K_{1}\right)$ with $\left(F+K_{1}\right) / K_{1}$. Hence $\left(F /\left(F \cap K_{1}\right)\right.$, $\left.\left\{\|\|_{p_{n}}\right\}\right)$ can be regarded as an $L^{\infty}$-m.n. subspace of $E / K_{1}$. We define a map $\psi: F /\left(F \cap K_{1}\right) \rightarrow B(H)$ by $\psi(\bar{x})=\phi(x)$ for $\bar{x} \in F /\left(F \cap K_{1}\right)$. The map $\psi$ is well-defined since the kernel of $\phi$ contains $F \cap K_{1}$. For each $\left[\bar{x}_{i j}\right] \in$ $M_{n}\left(F /\left(F \cap K_{1}\right)\right)$, we have

$$
\left\|\psi_{n}\left(\left[\bar{x}_{i j}\right]\right)\right\|_{n}=\left\|\phi_{n}\left(\left[x_{i j}\right]\right)\right\|_{n} \leq p_{n}\left(\left[x_{i j}\right]\right)=\left\|\left[\bar{x}_{i j}\right]\right\|_{p_{n}} .
$$

Hence $\psi: F /\left(F \cap K_{1}\right) \rightarrow B(H)$ is a complete contraction. By the ArvesonWittstock Hahn-Banach Theorem (cf. Theorem 4.2), there exists a completely contractive map $\bar{\psi}: E / K_{1} \rightarrow B(H)$, which extends $\psi$ and preserves the cbnorm. Let $\pi: E \rightarrow E / K_{1}$ be the natural quotient map and let $\bar{\phi}=\bar{\psi} \circ \pi: E \rightarrow$ $B(H)$. Then $\bar{\phi}$ extends $\phi$ and we have

$$
\left\|\bar{\phi}_{n}\left(\left[x_{i j}\right]\right)\right\|_{n}=\left\|\left(\bar{\psi}_{n} \circ \pi_{n}\right)\left(\left[x_{i j}\right]\right)\right\|_{n} \leq\left\|\left[\bar{x}_{i j}\right]\right\|_{p_{n}}=p_{n}\left(\left[x_{i j}\right]\right)
$$

for all $\left[x_{i j}\right] \in M_{n}(E)$. 


\section{Minimal $E$-PRojection of $B(H)$}

The main result (Theorem 3.4) of this section is to show that "For every operator space $E$ contained in $B(H)$ and a minimal $L^{\infty}$-matricial $E$ seminorm $\left\{p_{n}\right\}$ on $B(H)$, there exists a minimal $E$-projection $\phi$ of $B(H)$ with $\left\{p_{n}^{\phi}\right\}=\left\{p_{n}\right\}$ ". This result is motivated by Hamana [9] and will play a key role in the study of the injective envelopes of operator spaces.

Definition 3.1. Let $B(H)$ be the space of all bounded operators on a Hilbert space $H$. A linear map $\phi: B(H) \rightarrow B(H)$ is called a completely contractive projection if $\|\phi\|_{c b} \leq 1$ and $\phi^{2}=\phi$. Let $E$ be an operator space contained in $B(H)$. An E-projection of $B(H)$ is a completely contractive projection $\phi: B(H) \rightarrow B(H)$ such that $\phi(x)=x$ for all $x \in E$.

Let $\Xi_{B(H)}^{E}$ be the set of all $E$-projections of $B(H)$. Then $\Xi_{B(H)}^{E}$ is nonempty. Define a partial ordering on $\Xi_{B(H)}^{E}$ by saying

$$
\psi \lesssim \phi \text { if and only if } \psi \circ \phi=\phi \circ \psi=\psi .
$$

$A$ minimal E-projection of $B(H)$ is an $E$-projection of $B(H)$ which is minimal under this partial ordering.

Definition 3.2. Let $E$ be an $L^{\infty}$-m.n. space and $F$ an $L^{\infty}$-m.n. subspace of $E$. An $L^{\infty}$-matricial seminorm $\left\{p_{n}\right\}$ on $E$ is called an $L^{\infty}$-matricial $F$-seminorm if it satisfies:

$$
\begin{aligned}
& p_{n}(x) \leq\|x\|_{n} \text { for all } x \in M_{n}(E), \\
& p_{n}(x)=\|x\|_{n} \text { for all } x \in M_{n}(F) .
\end{aligned}
$$

Let $\Gamma_{E}^{F}$ be the set of all $L^{\infty}$-matricial $F$-seminorms on $E$. Then $\Gamma_{E}^{F}$ is nonempty. Define a partial ordering on $\Gamma_{E}^{F}$ by saying

$$
\left\{p_{n}\right\} \lesssim\left\{q_{n}\right\} \text { if and only if } p_{n}(x) \leq q_{n}(x)
$$

for all $x \in M_{n}(E)$ and $n \in N$.

Let $E$ be an operator space contained in $B(H)$ and $\phi: B(H) \rightarrow B(H)$ a complete contraction such that $\phi(x)=x$ for all $x \in E$. Then the matricial seminorms $\left\{p_{n}^{\phi}\right\}$ and $\left\{q_{n}^{\phi}\right\}$ in Example 2.3 and Example 2.4 are $L^{\infty}$-matricial $E$-seminorms on $B(H)$ and it is easy to see that $\left\{q_{n}^{\phi}\right\} \lesssim\left\{p_{n}^{\phi}\right\}$.

Proposition 3.3. The partially ordered set $\Gamma_{E}^{F}$ must have at least one minimal element.

Proof. Suppose that $\left\{\left\{p_{n}^{\gamma}\right\}_{n=1}^{\infty}\right\}_{\gamma \in \Lambda}$ is an arbitrary chain in $\Gamma_{E}^{F}$. Consider a function $p_{n}$ on $M_{n}(E)$ defined by

$$
p_{n}(x)=\inf \left\{p_{n}^{\gamma}(x) ; \gamma \in \Lambda\right\}
$$


for $x \in M_{n}(E)$. It is easy to see that for each $n \in N, p_{n}$ is a seminorm on $M_{n}(E)$ which satisfies (I) and (II) in Definition 3.2. For $x \in M_{n}(E)$ and $y \in M_{m}(E)$, we have

$$
\begin{aligned}
p_{n+m}(x \oplus y) & =\inf \left\{p_{n+m}^{\gamma}(x \oplus y) ; \gamma \in \Lambda\right\} \\
& =\inf \left\{\max \left\{p_{n}^{\gamma}(x), p_{m}^{\gamma}(y)\right\} ; \gamma \in \Lambda\right\} \\
& =\max \left\{\inf \left\{p_{n}^{\gamma}(x) ; \gamma \in \Lambda\right\}, \inf \left\{p_{m}^{\gamma}(y) ; \gamma \in \Lambda\right\}\right\} \\
& =\max \left\{p_{n}(x), p_{m}(y)\right\} .
\end{aligned}
$$

For $\alpha \in M_{n}$ and $x \in M_{n}(E)$, we have

$$
\begin{aligned}
p_{n}(\alpha x) & =\inf \left\{p_{n}^{\gamma}(\alpha x) ; \gamma \in \Lambda\right\} \\
& \leq\|\alpha\| \inf \left\{p_{n}^{\gamma}(x) ; \gamma \in \Lambda\right\} \\
& =\|\alpha\| p_{n}(x) .
\end{aligned}
$$

Similarly, we have $p_{n}(x \alpha) \leq\|\alpha\| p_{n}(x)$. Hence $\left\{p_{n}\right\}$ is an $L^{\infty}$-matricial $F$ seminorm on $E$ such that $\left\{p_{n}\right\} \lesssim\left\{p_{n}^{\gamma}\right\}$ for all $\gamma \in \Lambda$. By Zorn's lemma, the partially ordered set $\Gamma_{E}^{F}$ must have a minimal element.

A minimal element in the partially ordered set $\Gamma_{E}^{F}$ is called a minimal $L^{\infty}$. matricial F-seminorm on $E$.

Theorem 3.4. Let $E$ be an operator space contained in $B(H)$. For every minimal $L^{\infty}$-matricial E-seminorm $\left\{p_{n}\right\}$ on $B(H)$, there is a minimal E-projection $\phi$ of $B(H)$ with $\left\{p_{n}^{\phi}\right\}=\left\{p_{n}\right\}$.

Proof. Let id: $E \rightarrow B(H)$ be the inclusion map. Then we have

$$
\left\|\operatorname{id}_{n}(x)\right\|_{n}=\|x\|_{n}=p_{n}(x)
$$

for all $x \in M_{n}(E)$ and $n \in N$. By Theorem 2.5, there is a linear map $\phi: B(H) \rightarrow B(H)$ which extends id such that

$$
\left\|\phi_{n}(x)\right\|_{n} \leq p_{n}(x) \leq\|x\|_{n}
$$

for all $x \in M_{n}(B(H))$ and $n \in N$. Then $\left\{p_{n}^{\phi}\right\}$ and $\left\{q_{n}^{\phi}\right\}$ are $L^{\infty}$-matricial $E$-seminorms on $B(H)$ such that

$$
\left\{q_{n}^{\phi}\right\} \lesssim\left\{p_{n}^{\phi}\right\} \lesssim\left\{p_{n}\right\} .
$$

This implies that $\left\{q_{n}^{\phi}\right\}=\left\{p_{n}^{\phi}\right\}=\left\{p_{n}\right\}$ since $\left\{p_{n}\right\}$ is a minimal element in $\Gamma_{B(H)}^{E}$. 
Next we want to show that $\phi$ is a minimal $E$-projection of $B(H)$. Since

$$
\begin{aligned}
\left\|\phi(x)-\phi^{2}(x)\right\|_{1} & =\|\phi(x-\phi(x))\|_{1} \\
& =p_{1}^{\phi}(x-\phi(x))=q_{1}^{\phi}(x-\phi(x)) \\
& =\lim _{k \rightarrow \infty} \sup \left\|\left(\frac{\phi+\cdots+\phi^{k}}{k}\right)(x-\phi(x))\right\|_{1} \\
& =\lim _{k \rightarrow \infty}\left\|\frac{\phi(x)-\phi^{k+1}(x)}{k}\right\|_{1}=0
\end{aligned}
$$

for all $x \in B(H)$ we get $\phi^{2}=\phi$ and $\phi$ is an $E$-projection of $B(H)$. Let $\psi$ be an arbitrary $E$-projection of $B(H)$ with $\psi \lesssim \phi$. We have $\left\{q_{n}^{\psi}\right\}=\left\{p_{n}^{\phi}\right\}=\left\{p_{n}\right\}$. Then

$$
\begin{aligned}
\|\phi(x)-\psi(x)\|_{1} & =\|\phi(x)-\phi \circ \psi(x)\|_{1}=\|\phi(x-\psi(x))\|_{1} \\
& =p_{1}^{\phi}(x-\psi(x))=q_{1}^{\psi}(x-\psi(x))=0
\end{aligned}
$$

for all $x \in B(H)$. Therefore $\psi=\phi$ and $\phi$ is a minimal $E$-projection of $B(H)$ with $\left\{p_{n}^{\phi}\right\}=\left\{p_{n}\right\}$.

\section{INJECTIVE OPERATOR SPACES}

Definition 4.1. Let $E$ be an operator space. $E$ is called an injective operator space if for every operator space $F$, every operator subspace $F_{0}$ of $F$ and every completely bounded map $\phi: F_{0} \rightarrow E$, there exists a linear map $\bar{\phi}: F \rightarrow E$ such that $\left.\bar{\phi}\right|_{F_{0}}=\phi$ and $\|\bar{\phi}\|_{c b}=\|\phi\|_{c b}$.

The following theorem is due to Wittstock [22 and 23]. It is called the Arveson-Wittstock Hahn-Banach Theorem. Haagerup [8], Paulsen [15 and 16], and recently Effros-Ruan [6] have given different proofs of this result.

Theorem 4.2. Let $E$ be an operator space contained in a unital $C^{*}$-algebra $A$ and $\phi: E \rightarrow B(H)$ a complete contraction. Then there exists a complete contraction $\bar{\phi}: A \rightarrow B(H)$ such that $\left.\bar{\phi}\right|_{E}=\phi$ and $\|\bar{\phi}\|_{c b}=\|\phi\|_{c b}$.

Remark. The Arveson-Wittstock Hahn-Banach Theorem fails for general m.n. spaces. We have shown in [6] that there exist $L^{1}$-m.n. spaces $F \subseteq E$ and a completely bounded map $\phi: F \rightarrow M_{2}(C)$ so that $\phi$ cannot be extended to a completely bounded map $\hat{\phi}: E \rightarrow M_{2}(C)$ with $\|\phi\|_{c b}=\|\hat{\phi}\|_{c b}$.

From the Arveson-Wittstock Hahn-Banach Theorem, we know that $B(H)$ is an injective operator space for arbitrary Hilbert space $H$. Then an operator space $E$ contained in $B(H)$ is injective if and only if there exists a completely contractive projection $\phi$ from $B(H)$ onto $E$. This implies that injective operator spaces must be norm closed. Obviously, every injective $C^{*}$-algebra is an injective operator space. Furthermore if $A$ is an injective $C^{*}$-algebra and $p, q$ are projections in $A$, then $p A q$ is again an injective operator space. In 
particular, we know that $B(H, K)$ is an injective operator space, where $H$ and $K$ are Hilbert spaces. Our next theorem gives a negative answer to Wittstock's question [23, Problem 5.1].

Theorem 4.3. There exists an injective operator space $E$ which is not completely isometric to any $C^{*}$-algebra.

Proof. Let $E=\left\{\left[\begin{array}{ll}a & b \\ 0 & 0\end{array}\right], a, b \in C\right\}$. It is easy to see that $E$ is an injective operator space contained in $M_{2}(C)$. Consider $F=\left\{\left[\begin{array}{ll}a & 0 \\ b & 0\end{array}\right], a, b \in C\right\}$. Then $F$ is also an operator space. Let $\theta: F \rightarrow E$ be the transpose map defined by

$$
\theta\left(\left[\begin{array}{ll}
a & 0 \\
b & 0
\end{array}\right]\right)=\left[\begin{array}{ll}
a & b \\
0 & 0
\end{array}\right] .
$$

Then $\theta$ is an isometry. On the other hand, for

$$
\left[\begin{array}{cc}
E_{11} & E_{21} \\
0 & 0
\end{array}\right] \in M_{2}\left(M_{2}(C)\right)
$$

we have

but

$$
\left\|\left[\begin{array}{cc}
E_{11} & E_{21} \\
0 & 0
\end{array}\right]\right\|_{2}=1
$$

$$
\left\|\theta_{2}\left(\left[\begin{array}{cc}
E_{11} & E_{21} \\
0 & 0
\end{array}\right]\right)\right\|_{2}=\left\|\left[\begin{array}{cc}
E_{11} & E_{12} \\
0 & 0
\end{array}\right]\right\|_{2}=2^{1 / 2} .
$$

Therefore we get $\|\theta\|_{c b}=\left\|\theta_{2}\right\| \geq 2^{1 / 2}$ (cf. Smith [19]).

Suppose that $E$ is completely isometric to a $C^{*}$-algebra $A$. Since $\operatorname{dim} A=$ 2 , we must have that $A$ is "-isomorphic to $C \oplus C$. Thus $A$ is a commutative $C^{*}$-algebra and we have $\|\theta\|_{c b}=\|\theta\|=1$ (cf. Loebl [13, Lemma 1]), a contradiction. Thus $E$ cannot be completely isometric to any $C^{*}$-algebra.

If $E$ is an operator space contained in $B(H)$, then there exists a minimal $E$-projection of $B(H)$ (Theorem 3.4). The following lemma shows that if we choose such a minimal $E$-projection carefully, we can find two other unital completely positive projections $\psi_{i}: B(H) \rightarrow B(H)(i=1,2)$ so that the map

$$
\Phi=\left[\begin{array}{ll}
\psi_{1} & \phi \\
\phi^{*} & \psi_{2}
\end{array}\right]: M_{2}(B(H)) \rightarrow M_{2}(B(H))
$$

is a unital completely positive projection.

Lemma 4.4. Let $E$ be an operator space contained in $B(H)$. Then there exists a minimal E-projection $\phi$ of $B(H)$ and two unital completely positive projections $\psi_{i}(i=1,2)$ so that the map

$$
\Phi=\left[\begin{array}{ll}
\psi_{1} & \phi \\
\phi^{*} & \psi_{2}
\end{array}\right]
$$

is a unital completely positive projection.

Proof. Let $L^{E}=\left\{\left(\begin{array}{c}\lambda^{*} \\ y_{\mu}\end{array}\right) ; \lambda, \mu \in C, x, y \in E\right\}$. Then $L^{E}$ is an operator system contained in $M_{2}(B(H))$. We have an $L^{\infty}$-matricial $L^{E}$-seminorm on 
$M_{2}(B(H))$ which is minimal in the partially ordered set $\Gamma_{M_{2}(B(H))}^{L^{E}}$ (Proposition 3.3). Fixing such a minimal $L^{\infty}$-matricial $L^{E}$-seminorm $\left\{p_{n}\right\}$ on $M_{2}(B(H))$, there exists a minimal $L^{E}$-projection $\Phi$ of $M_{2}(B(H))$ such that $\left\{p_{n}^{\Phi}\right\}=\left\{p_{n}\right\}$ (Theorem 3.4). We know from Arveson [1] that $\Phi$ must be completely positive since $\Phi$ is a complete contraction with $\Phi\left(1_{2}\right)=1_{2}$. By Stinespring's Theorem [20], there exists a Hilbert space $\bar{K},{ }^{*}{ }^{*}$-representation $\bar{\pi}: M_{2}(B(H)) \rightarrow B(\bar{K})$ with $\bar{\pi}\left(1_{2}\right)=1_{\bar{K}}$ and an isometry $V: H \oplus H \rightarrow \bar{K}$ such that

$$
\Phi\left(\left[x_{i j}\right]\right)=V^{*} \bar{\pi}\left(\left[x_{i j}\right]\right) V
$$

for all $\left[x_{i j}\right] \in M_{2}(B(H))$.

Let $\left\{E_{i j}\right\}$ be the usual matrix units in $M_{2}(B(H))$. Then $\left\{\bar{\pi}\left(E_{i j}\right)\right\}$ are matrix units in $B(\bar{K})$ which give a decomposition of $\bar{K}$ into $K \oplus K$. If we write $\varepsilon_{i j}=\bar{\pi}\left(E_{i j}\right)$, then $\varepsilon_{11}$ is the projection onto $K \oplus 0$. Consider

$$
\pi(x)=\bar{\pi}\left(\left(\begin{array}{ll}
x & 0 \\
0 & 0
\end{array}\right)\right)=\varepsilon_{11} \bar{\pi}(x \oplus x) \varepsilon_{11}
$$

for all $x \in B(H)$. Then $\pi: B(H) \rightarrow B(K)$ is a *-representation with $\pi(1)=1_{K}$ and we have $\bar{\pi}=\pi \otimes \mathrm{id}_{2}$. Following the proof in [16, Theorem 2.7], we have $V\left(\xi_{1} \oplus \xi_{2}\right)=V_{1} \xi_{1} \oplus V_{2} \xi_{2}$ for some isometries $V_{i}: H \rightarrow K(i=1,2)$ and

$$
\begin{aligned}
\Phi\left(\left[x_{i j}\right]\right) & =V^{*}\left(\pi \otimes \mathrm{id}_{2}\right)\left(\left[x_{i j}\right]\right) V \\
& =\left[\begin{array}{cc}
V_{1}^{*} & 0 \\
0 & V_{2}^{*}
\end{array}\right]\left[\begin{array}{ll}
\pi\left(x_{11}\right) & \pi\left(x_{12}\right) \\
\pi\left(x_{21}\right) & \pi\left(x_{22}\right)
\end{array}\right]\left[\begin{array}{cc}
V_{1} & 0 \\
0 & V_{2}
\end{array}\right] \\
& =\left[\begin{array}{cc}
V_{1}^{*} \pi\left(x_{11}\right) V_{1} & V_{1}^{*} \pi\left(x_{12}\right) V_{2} \\
V_{2}^{*} \pi\left(x_{21}\right) V_{1} & V_{2}^{*} \pi\left(x_{22}\right) V_{2}
\end{array}\right]
\end{aligned}
$$

for all $\left[x_{i j}\right] \in M_{2}(B(H))$. Therefore, we have

$$
\Phi=\left[\begin{array}{ll}
\psi_{1} & \phi \\
\phi^{*} & \psi_{2}
\end{array}\right]
$$

where $\phi=V_{1}^{*} \pi V_{2}$ is an $E$-projection of $B(H)$ and $\psi_{i}=V_{i}^{*} \pi V_{i}(i=1,2)$ are unital completely positive projections from $B(H)$ into $B(H)$.

We need to show that $\phi$ is a minimal $E$-projection of $B(H)$. Let $E^{\phi}=$ $\phi(B(H))$. Then $E^{\phi}$ is an injective operator space contained in $B(H)$. Suppose that $\phi_{0}$ is another $E$-projection of $B(H)$ such that $\phi_{0} \circ \phi=\phi \circ \phi_{0}=\phi_{0}$. Then the injective operator space $E^{\phi_{0}}$ is contained in $E^{\phi}$. Assume that $\phi_{0} \neq \phi$. Then $E^{\phi_{0}}$ is a proper subspace of $E^{\phi}$. The identity map id: $E^{\phi_{0}} \rightarrow E^{\phi_{0}}$ can be extended to a complete contraction $T: E^{\phi} \rightarrow E^{\phi_{0}}$. Hence there exists an element $x_{0} \neq 0 \in \operatorname{ker} T$.

Let $L^{E^{\prime \prime}}=\left\{\left(\begin{array}{c}\lambda \\ y^{*}\end{array}\right) ; \lambda, \mu \in C, x, y \in E^{\phi}\right\}$. Then $L^{E^{\prime \prime}}$ is an operator system contained in $M_{2}(B(H))$ and we consider a map $\Psi: L^{E^{\prime \prime}} \rightarrow M_{2}(B(H))$ defined by

$$
\Psi\left(\left[\begin{array}{cc}
\lambda & x \\
y^{*} & \mu
\end{array}\right]\right)=\left[\begin{array}{cc}
\lambda & T(x) \\
(T(y))^{*} & \mu
\end{array}\right] .
$$


Then $\Psi$ is a unital completely positive map since $T$ is a complete contraction (Paulsen [17, Lemma 7.1]). Therefore we have

$$
\left\|\Psi_{n}\left(\left[u_{i j}\right]\right)\right\|_{n} \leq\left\|\left[u_{i j}\right]\right\|_{n}=\left\|\Phi_{n}\left(\left[u_{i j}\right]\right)\right\|_{n}=p_{n}\left(\left[u_{i j}\right]\right)
$$

for all $\left[u_{i j}\right] \in M_{n}\left(L^{E^{\phi}}\right)$. We can extend $\Psi$ to $\bar{\Psi}: M_{2}(B(H)) \rightarrow M_{2}(B(H))$ such that

$$
\left\|\bar{\Psi}_{n}\left(\left[u_{i j}\right]\right)\right\|_{n} \leq p_{n}\left(\left[u_{i j}\right]\right)
$$

for all $\left[u_{i j}\right] \in M_{n}\left(M_{2}(B(H))\right)$ and $n \in N$. Then $\left\{p_{n}^{\bar{\Psi}}\right\}$ is an $L^{\infty}$-matricial $L^{E}$-seminorm on $M_{2}(B(H))$ such that $\left\{p_{n}^{\bar{\Psi}}\right\} \lesssim\left\{p_{n}\right\}$. It follows from the assumption that

and

$$
p_{1}^{\bar{\Psi}}\left(\left[\begin{array}{cc}
0 & x_{0} \\
x_{0}^{*} & 0
\end{array}\right]\right)=0
$$

$$
\begin{aligned}
p_{1}\left(\left[\begin{array}{cc}
0 & x_{0} \\
x_{0}^{*} & 0
\end{array}\right]\right) & =\left\|\Phi\left(\left[\begin{array}{cc}
0 & x_{0} \\
x_{0}^{*} & 0
\end{array}\right]\right)\right\|_{1} \\
& =\left\|\left[\begin{array}{cc}
0 & x_{0} \\
x_{0}^{*} & 0
\end{array}\right]\right\|_{1}=\left\|x_{0}\right\| \neq 0 .
\end{aligned}
$$

Then $\left\{p_{n}^{\bar{\Psi}}\right\} \neq\left\{p_{n}\right\}$. This contradicts the minimality of $\left\{p_{n}\right\}$. Therefore we must have $\phi_{0}=\phi$ and $\phi$ is a minimal $E$-projection.

In the following theorem, we study the structures of injective operator spaces.

Theorem 4.5. Let $E$ be an operator space. Then $T F A E$ :

(1) $E$ is an injective operator space;

(2) there exists an injective $C^{*}$-algebra $A$ and two projections $p, q \in A$ such that $E$ is completely isometric to $p A q$.

Proof. (2) $\Rightarrow(1)$ is trivial.

$(1) \Rightarrow(2)$ We may assume that $E$ is an injective operator space contained in $B(H)$. Using the same notations as in Lemma 4.4, we have a minimal $E$ projection $\phi$ of $B(H)$ and two unital completely contractive projections $\psi_{i}$ $(i=1,2)$ from $B(H)$ into $B(H)$ such that the map

$$
\Phi=\left[\begin{array}{ll}
\psi_{1} & \phi \\
\phi^{*} & \psi_{2}
\end{array}\right]: M_{2}(B(H)) \rightarrow M_{2}(B(H))
$$

is a unital completely positive projection. Then

$$
A=\Phi\left(M_{2}(B(H))\right) \subseteq M_{2}(B(H))
$$

with the multiplication defined by $x \cdot y=\Phi(x y)$ for all $x, y \in A$ is an injective $C^{*}$-algebra (cf. Choi and Effros [4]).

Consider $p=\Phi\left(E_{11}\right)$ and $q=\Phi\left(E_{22}\right)$. Obviously, $p$ and $q$ are two projections in $A$. Since $E$ is an injective operator space and $\phi$ is a minimal $E$-projection, we must have $E=E^{\phi}$. Define a map $T: E \rightarrow p A q$ by

$$
T(x)=p \cdot\left[\begin{array}{ll}
0 & x \\
0 & 0
\end{array}\right] \cdot q=\left[\begin{array}{ll}
0 & x \\
0 & 0
\end{array}\right]
$$


for all $x \in E \subseteq B(H)$. Thus $T$ is a well-defined complete isometry. Notice that

$$
p \cdot A \cdot q=\Phi\left(E_{11} M_{2}(B(H)) E_{22}\right)=\Phi\left(\left[\begin{array}{cc}
0 & B(H) \\
0 & 0
\end{array}\right]\right)=\left[\begin{array}{cc}
0 & E^{\phi} \\
0 & 0
\end{array}\right] .
$$

This implies that $T$ is onto. Deleting the dot notation for the product in $A$, we are done.

\section{INJECTIVE ENVELOPES OF OPERATOR SPACES}

Definition 5.1. Let $E$ be an operator space. An extension of $E$ is a pair $(Z, \kappa)$ of an operator space $Z$ and a completely isometric embedding $\kappa: E \rightarrow Z$. An extension $(Z, \kappa)$ of $E$ is called injective if $Z$ is an injective operator space. An extension $(Z, \kappa)$ of $E$ is called an injective envelope of $E$ if $(Z, \kappa)$ is an injective extension of $E$ and $\mathrm{id}_{Z}$ is the only complete contraction (from $Z$ into $Z$ ) which extends $\operatorname{id}_{\kappa(E)}: \kappa(E) \rightarrow Z$ from $\kappa(E)$ to $Z$.

Remark. In the category of Banach spaces and contractive linear maps, the existence and uniqueness of injective envelopes of Banach spaces were studied by Cohen [4]. The original definition of injective envelopes of Banach spaces is as follows:

Let $E$ be a Banach space. An injective envelope of $E$ is a pair $(Z, \kappa)$ of an injective Banach space $Z$ and an isometric embedding $\kappa: E \rightarrow Z$ such that the only injective subspace of $Z$ containing $\kappa(E)$ is $Z$ itself.

Isbell [10] pointed out that this definition is equivalent to the following one:

Let $E$ be a Banach space. An injective envelope of $E$ is a pair $(Z, \kappa)$ of an injective Banach space $Z$ and an isometric embedding $\kappa: E \rightarrow Z$ such that $\mathrm{id}_{Z}$ is the only contractive extension of $\mathrm{id}_{\kappa(E)}: \kappa(E) \rightarrow Z$ from $\kappa(E)$ to $Z$.

Our definition of injective envelopes of operator spaces is an analogue of the second one (see, however, Theorem 5.6). Most of this section is inspired by Hamana [8], where he studied the injective envelopes of unital $C^{*}$-algebras.

Theorem 5.2. Every operator space has an injective envelope.

Proof. Assume that $E$ is an operator space contained in $B(H)$. Then there exists a minimal $L^{\infty}$-matricial $E$-seminorm $\left\{p_{n}\right\}$ on $B(H)$ and a minimal $E$-projection $\phi$ of $B(H)$ such that $\left\{p_{n}^{\phi}\right\}=\left\{p_{n}\right\}$. Then $E^{\phi}=\phi(B(H))$ is an injective operator space containing $E$ as a subspace. The pair $\left(E^{\phi}, \mathrm{id}_{E}\right)$ is an injective extension of $E$, where $\mathrm{id}_{E}: E \rightarrow E^{\phi}$ is the natural embedding map. Let $\psi: E^{\phi} \rightarrow E^{\phi}$ be a completely contractive extension of $\mathrm{id}_{E}$. Then

$$
\left\|\psi_{n}(x)\right\|_{n} \leq\|x\|_{n}=\left\|\phi_{n}(x)\right\|_{n}=p_{n}(x)
$$

for all $x \in M_{n}\left(E^{\phi}\right)$. We can extend $\psi$ again to a complete contraction $\bar{\psi}: B(H) \rightarrow E^{\phi}$ such that $\left\|\bar{\psi}_{n}(x)\right\| \leq p_{n}(x)$ for all $x \in M_{n}(B(H))$. Then $\left\{q_{n}^{\bar{\psi}}\right\}$ is an $L^{\infty}$-matricial $E$-seminorm on $B(H)$ such that

$$
\left\{q_{n}^{\bar{\psi}}\right\} \lesssim\left\{p_{n}\right\} \text {. }
$$


Hence we must have $\left\{q_{n}^{\bar{\psi}}\right\}=\left\{p_{n}\right\}$ since $\left\{p_{n}\right\}$ is a minimal $L^{\infty}$-matricial $E$ projection. Now for every $x \in E^{\phi}$, we have

$$
\|x-\psi(x)\|_{1}=p_{1}(x-\bar{\psi}(x))=q_{1}^{\bar{\psi}}(x-\bar{\psi}(x))=0 .
$$

Therefore $\psi=\mathrm{id}_{E^{\phi}}$ and $\left(E^{\phi}, \kappa\right)$ is an injective envelope of $E$.

Proposition 5.3. Let $E_{1}, E_{2}$ be two operator spaces and $\left(Z_{1}, \kappa_{1}\right)$ and $\left(Z_{2}, \kappa_{2}\right)$ injective envelopes of $E_{1}, E_{2}$, respectively. Then for every complete isometry $\lambda$ from $E_{1}$ onto $E_{2}$, there exists a unique complete isometry $\hat{\lambda}$ from $Z_{1}$ onto $Z_{2}$ such that

$$
\hat{\lambda} \circ \kappa_{1}=\kappa_{2} \circ \lambda \text {. }
$$

Proof. Let $\lambda: E_{1} \rightarrow E_{2}$ be a complete isometry from $E_{1}$ onto $E_{2}$. Then

$$
\mu=\kappa_{2} \circ \lambda \circ \kappa_{1}^{-1}: \kappa_{1}\left(E_{1}\right) \rightarrow \kappa_{2}\left(E_{2}\right)
$$

is a complete isometry from $\kappa_{1}\left(E_{1}\right)$ onto $\kappa_{2}\left(E_{2}\right) . \mu$ can be extended to a complete contraction $\hat{\lambda}: Z_{1} \rightarrow Z_{2}$. Since $\mu^{-1}: \kappa_{2}\left(E_{2}\right) \rightarrow \kappa_{1}\left(E_{1}\right)$ is also a complete isometry, $\mu^{-1}$ can be extended to a complete contraction $\hat{\mu}: Z_{2} \rightarrow$ $Z_{1}$.

Then $\hat{\mu} \circ \hat{\lambda}: Z_{1} \rightarrow Z_{1}$ is a complete contraction such that $\left.\hat{\mu} \circ \hat{\lambda}\right|_{\kappa_{1}\left(E_{1}\right)}=\mathrm{id}_{\kappa_{1}\left(E_{1}\right)}$. Hence $\hat{\mu} \circ \hat{\lambda}=\mathrm{id}_{Z_{1}}$. Similarly we have $\hat{\lambda} \circ \hat{\mu}=\mathrm{id}_{Z_{2}}$. This implies that $\hat{\lambda}$ is a complete isometry from $Z_{1}$ onto $Z_{2}$ and $\hat{\lambda} \circ \kappa_{1}=\kappa_{2} \circ \lambda$.

If we have another complete isometry $\lambda^{*}$ from $Z_{1}$ onto $Z_{2}$ such that $\lambda^{*}$ 。 $\kappa_{1}=\kappa_{2} \circ \lambda$, it follows easily from the above arguments that $\lambda^{*}=\hat{\lambda}$.

Theorem 5.4. Let $E$ be an operator space. If $\left(Z_{1}, \kappa_{1}\right)$ and $\left(Z_{2}, \kappa_{2}\right)$ are two injective envelopes of $E$, then there exists a unique complete isometry $\hat{\lambda}$ from $Z_{1}$ onto $Z_{2}$ such that $\hat{\lambda} \circ \kappa_{1}=\kappa_{2}$. Hence every operator space $E$ has an essentially unique injective envelope.

Proof. Let $\lambda=\kappa_{2} \circ \kappa_{1}^{-1}: \kappa_{1}(E) \rightarrow \kappa_{2}(E)$. Then $\lambda$ is a complete isometry from $\kappa_{1}(E)$ onto $\kappa_{2}(E)$ and $\lambda$ can be extended uniquely to a complete isometry $\hat{\lambda}$ from $Z_{1}$ onto $Z_{2}$.

Lemma 5.5. Let $E \subseteq Z \subseteq B(H)$ and $\left(Z, \mathrm{id}_{E}\right)$ an injective extension of $E$. Then $\left(Z, \mathrm{id}_{E}\right)$ is an injective envelope of $E$ if and only if there exists a minimal $E$-projec-

tion $\phi$ of $B(H)$ such that $Z=E^{\phi}=\phi(B(H))$.

Proof. $\Rightarrow$ Since $Z$ is an injective subspace of $B(H)$, there exists a $Z$-projection $\phi$ of $B(H)$ such that $E^{\phi}=\phi(B(H))=Z$. Then $\phi$ is also an $E$-projection of $B(H)$.

Let $\psi$ be a minimal $E$-projection of $B(H)$ such that $\psi \lesssim \phi$, i.e. $\phi \circ \psi=$ $\psi \circ \phi=\psi$. Then $E^{\psi} \subseteq E^{\phi}$ and $E^{\psi}$ is an injective operator space containing $E$ as a subspace. The identity map $\mathrm{id}_{E^{\psi}}: E^{\psi} \rightarrow E^{\psi}$ can be extended to a complete 
contraction $\lambda: E^{\phi} \rightarrow E^{\psi}$. Thus $\lambda: E^{\phi} \rightarrow E^{\psi} \subseteq E^{\phi}$ is a complete contraction such that $\left.\lambda\right|_{E}=\mathrm{id}_{E}$. Hence we have $\lambda=\mathrm{id}_{E^{\phi}}$ and $E^{\psi}=E^{\phi}$. This implies that $\psi=\phi$ and $\phi$ is a minimal $E$-projection of $B(H)$.

$\Leftarrow$ Suppose that $\phi$ is a minimal $E$-projection of $B(H)$ such that $Z=$ $\phi(B(H))$. Let $\lambda: Z \rightarrow Z$ be a complete contraction such that $\left.\lambda\right|_{E}=\mathrm{id}_{E}$. We want to show that $\lambda=\mathrm{id}_{Z}$. Obviously, $\left\{q_{n}^{\lambda}\right\}$ is an $L^{\infty}$-matricial $E$-seminorm on $Z$ such that

$$
\left\{q_{n}^{\lambda}\right\} \lesssim\left\{p_{n}^{\phi}\right\}=\left\{\|\|_{n}\right\} .
$$

Following the proofs of Proposition 3.3 and Theorem 3.4, we can show that there exists a minimal $L^{\infty}$-matricial $E$-seminorm $\left\{p_{n}\right\}$ on $Z$ such that $\left\{p_{n}\right\} \lesssim$ $\left\{q_{n}^{\lambda}\right\}$ and a minimal $E$-projection $\psi$ of $Z$ such that $\left\{p_{n}^{\psi}\right\}=\left\{p_{n}\right\}$.

Let $\bar{\psi}=\psi \circ \phi$. Then $\bar{\psi}$ is an $E$-projection of $B(H)$ and $\bar{\psi} \lesssim \phi$. Thus we have $\bar{\psi}=\phi$ since $\phi$ is a minimal $E$-projection of $B(H)$. This implies that $p_{1}(x)=p_{1}^{\psi}(x)=\|x\|_{1}$ and then $\|x\|_{1}=q_{1}^{\lambda}(x)$ for all $x \in Z$. Therefore we have $\lambda=\mathrm{id}_{Z}$ since $\|x-\lambda(x)\|_{1}=q_{1}^{\lambda}(x-\lambda(x))=0$ for all $x \in Z$.

Remark. Let $E$ be an operator space containing 1 in $B(H)$ and $(Z, \kappa)$ an injective envelope of $E$. Then $Z$ is completely isometric to a injective $C^{*}$ algebra. To see this, we assume that $1 \in E \subseteq B(H)$ and $\phi: B(H) \rightarrow B(H)$ is a minimal $E$-projection of $B(H)$. Then $\left(E^{\phi}, \mathrm{id}_{E}\right)$ is completely isometric to $(Z, \kappa)$ and $E^{\phi}$ is an injective operator system, which is completely order isomorphic to a unital injective $C^{*}$-algebra (cf. Choi and Effros [4]). In particular, if $A$ is a unital $C^{*}$-algebra and $(B, \kappa)$ is an injective envelope of $A$ with $\kappa(1)=1$, then $(B, \kappa)$ is also an injective envelope of $A$ in the category of unital $C^{*}$-algebras and unital completely positive maps (cf. Hamana [8]).

The following theorem will show that the injective envelopes of operator spaces are the smallest injective extensions of $E$.

Theorem 5.6. Let $(Z, \kappa)$ be an injective extension of $E$. Then $(Z, \kappa)$ is an injective envelope of $E$ if and only if the only injective subspace of $Z$ containing $\kappa(E)$ is $Z$ itself, i.e. if there exists an injective subspace $Z_{1}$ of $Z$ containing $\kappa(E)$ then $Z_{1}=Z$.

Proof. $\Rightarrow$ Without loss of generality, we may assume that $E$ is a subspace of $Z$ and $\kappa=\mathrm{id}_{E}$ is the inclusion map from $E$ into $Z$. If $Z_{1}$ is an injective operator subspace of $Z$ and $E$ is contained in $Z_{1}$, then the identity map $\operatorname{id}_{Z_{1}}: Z_{1} \rightarrow Z_{1}$ can be extended to a complete contraction $\lambda: Z \rightarrow Z_{1} \subseteq Z$ such that $\left.\lambda\right|_{E}=\mathrm{id}_{E}$. Hence we must have that $\lambda=\mathrm{id}_{Z}$ and $Z_{1}=Z$.

$\Leftarrow$ Assume that $E \subseteq Z \subseteq B(H)$. The identity map $\operatorname{id}_{Z}: Z \rightarrow Z$ can be extended to a complete contraction $\phi: B(H) \rightarrow Z \subseteq B(H)$. Then $\phi$ is an $E$ projection of $B(H)$ such that $Z=E^{\phi}$. If we have another $E$-projection $\psi$ of $B(H)$ such that $\psi \circ \phi=\phi \circ \psi=\psi$, we have $E \subseteq E^{\psi} \subseteq E^{\phi}$ and $E^{\psi}$ is injective. Then we have $E^{\psi}=E^{\phi}$ and $\psi=\phi$. Hence $\phi$ is a minimal $E$-projection of $B(H)$ and $(Z, \kappa)$ is an injective envelope of $E$ by Lemma 5.5. 
Definition 5.7. Let $E$ be an operator space. An extension $(Z, \kappa)$ of $E$ is called an essential extension if for any operator space $F$ and a complete contraction $\phi: Z \rightarrow F, \phi$ is a complete isometry if $\phi \circ \kappa$ is a complete isometry.

Lemma 5.8. Let $(Z, \kappa)$ be an injective envelope of an operator space $E$. Then $(Z, \kappa)$ is an essential extension of $E$.

Proof. Assume that $E$ is contained in $Z$ and $\kappa=\mathrm{id}_{E}$. Suppose that $F$ is an operator space and $\phi: Z \rightarrow F$ is a complete contraction such that $\phi \circ \mathrm{id}_{E}$ is a complete isometry. Then $\phi^{-1}: \phi(E) \rightarrow Z$ is a complete isometry which can be extended to a complete contraction $\psi: F \rightarrow Z$. Then $\psi \circ \phi: Z \rightarrow Z$ is a complete contraction such that $\left.\psi \circ \phi\right|_{E}=\mathrm{id}_{E}$. Hence $\psi \circ \phi=\mathrm{id}_{Z}$ since $Z$ is an injective envelope of $E$. This implies that $\phi$ must be a complete isometry.

Theorem 5.9. Let $(Z, \kappa)$ be an extension of an operator space $E$. Then $(Z, \kappa)$ is an injective envelope of $E$ if and only if it is an injective and essential extension of $E$.

Proof. $\Rightarrow$ follows from Lemma 5.8.

$\Leftarrow$ Let $(\widehat{Z}, \hat{\kappa})$ be an injective envelope of $E$ and $\lambda: Z \rightarrow \widehat{Z}$ a completely contractive extension of $\hat{\kappa} \circ \kappa^{-1}: \kappa(E) \rightarrow \hat{Z}$. Then $\lambda$ is a complete isometry since $Z$ is an essential extension of $E$ with $\lambda \circ \kappa=\hat{\kappa}$. This implies that $(\lambda(Z), \hat{\kappa})$ is an injective extension of $E$ which is contained in the injective envelope of $(\hat{Z}, \hat{\kappa})$. From Theorem 5.6, we have that $\lambda(Z)=\hat{Z}$ and then $(Z, \kappa)$ is an injective envelope of $E$.

Corollary 5.10. Let $(\widehat{Z}, \hat{\kappa})$ be an injective envelope of an operator space $E$. Then for every essential extension $(Z, \kappa)$ of $E$, there exists a complete isometry $\lambda: Z \rightarrow \hat{Z}$ such that $\lambda \circ \kappa=\hat{\kappa}$. Hence the injective envelope of an operator space $E$ is a "maximal" essential extension of $E$.

Proof. The first statement of this corollary follows easily from the proof of Theorem 5.9. The proof of the second statement is trivial.

Remark. Masamichi Hamana has kindly informed the author that he has independently obtained Theorem 4.5 and Theorem 5.4 of this paper in connection to his earlier work: Injective envelopes of operator systems, Publ. Res. Inst. Math. Sci. 15 (1979), 773-785.

\section{REFERENCES}

1. W. B. Arveson, Subspaces of $C^{*}$-algebras. I, Acta Math. 123 (1969), 141-224.

2. W. G. Bade, The Banach space $C(S)$, Aarhus Univ. Lecture Notes Series No. 26, 1971.

3. M. D. Choi and E. G. Effros, Injectivity and operator spaces, J. Funct. Anal. 24 (1977), 156209.

4. H. B. Cohen, Injective envelope of Banach spaces, Bull. Amer. Math. Soc. 70 (1964), 723-726.

5. E. G. Effros, On multilinear completely bounded module maps, Contemp. Math. 62 (1987), 479-501. 
6. E. G. Effros and Z.-J. Ruan, On matricially normed spaces, Pacific J. Math 132 (1988), 243264.

7. D. B. Goodner, Projections in normed linear spaces, Trans. Amer. Math. Soc. 35 (1950), 89108.

8. U. Haagerup, Decomposition of completely bounded maps on operator algebras (unpublished), 1980.

9. M. Hamana, Injective envelopes of $C^{*}$-algebras, J. Math. Soc. Japan 31 (1979), 181-197.

10. M. Hasumi, The extension property of complex Banach spaces, Tôhoku Math. J. 10 (1958), 135-142.

11. J. R. Isbell, Three remarks on injective envelopes of Banach spaces, J. Math. Anal. Appl. 27 (1969), 516-518.

12. J. L. Kelley, Banach spaces with the extension property, Trans. Amer. Math. Soc. 72 (1952), 323-326.

13. R. I. Loebl, Contractive linear maps on $C^{*}$-algebras, Michigan Math. J. 22 (1975), 361-366.

14. L. Nachbin, A theorem of the Hahn-Banach type for linear transformations, Trans. Amer. Math. Soc. 68 (1950), 28-46.

15. V. I. Paulsen, Completely bounded maps on $C^{*}$-algebras and invariant operator ranges, Proc. Amer. Math. Soc. 86 (1982), 91-96.

16. _ Every completely polynomially bounded operator is similar to a contraction, J. Funct. Anal. 55 (1984), 1-17.

17. Completely bounded maps and dilations, Pitman Research Notes in Mathematics Series, 146, Wiley, New York, 1986.

18. Z.-J. Ruan, Subspaces of $C^{*}$-algebras, J. Funct. Anal. 76 (1988), 217-230.

19. R. R. Smith, Completely bounded maps between $C^{*}$-algebras, J. London Math. Soc. (2) 27 (1983), 157-166.

20. W. F. Stinespring, Positive functions on $C^{*}$-algebras, Proc. Amer. Math. Soc. 6 (1955), $211-$ 216.

21. J. Tomiyama, On the transpose map of matrix algebras, Proc. Amer. Math. Soc. 88 (1983), 635-638.

22. G. Wittstock, Ein operatorwertiger Hahn-Banach Satz, J. Funct. Anal. 40 (1981), 127-150.

23. __ Extensions of completely bounded $C^{*}$-module homomorphisms, Proc. Conference on Operator Algebras and Group Representations (Neptun, 1980), Pitman, 1984.

24. M. A. Youngson, Completely contractive projections on $C^{*}$-algebras, Quart. J. Math. Oxford Ser (2) 34 (1983), 50்7-511.

Department of Mathematics, University of California, Berkeley, California 94720

DEPARTMENT OF MATHEMATICS, UNIVERsity OF IlLiNois, Urbana, ILLINOIS 61801 